\title{
miR-3940-5p/miR-8069 ratio in urine exosomes is a novel diagnostic biomarker for pancreatic ductal adenocarcinoma
}

\author{
NAOHIKO YOSHIZAWA ${ }^{1}$, KAZUSHI SUGIMOTO ${ }^{1,2}$, MASAHIKO TAMEDA $^{1}$, YUJI INAGAKI ${ }^{1}$, \\ MAKOTO IKEJIRI $^{2}$, HIROYUKI INOUE ${ }^{1}$, MASANOBU USUI ${ }^{3}$, MASAAKI ITO $^{4}$ and YOSHIYUKI TAKEI ${ }^{1}$ \\ ${ }^{1}$ Department of Gastroenterology and Hepatology, Mie University Graduate School of Medicine; ${ }^{2}$ Department of \\ Central Laboratory, Mie University Hospital; Departments of ${ }^{3}$ Hepatobiliary Pancreatic and Transplant Surgery, \\ and ${ }^{4}$ Cardiology and Nephrology, Mie University Graduate School of Medicine, Tsu, Mie 514-8507, Japan
}

Received July 16, 2019; Accepted November 11, 2019

DOI: $10.3892 / 01.2020 .11357$

\begin{abstract}
Despite the development of several therapeutic options, the prognosis of pancreatic cancer remains poor. One reason for this is the difficulty of diagnosing the disease at an early stage. For example, carbohydrate antigen (CA) 19-9, which is the most widely used biomarker for pancreatic cancer, cannot be used to detect the disease at early stages. Some studies have attempted to find novel biomarkers for pancreatic cancer. The aim of the present study was to find a novel diagnostic biomarker for pancreatic ductal adenocarcinoma (PDAC) in urine exosomes. Exosomes were isolated from urine and serum samples of patients with PDAC and control subjects, or culture media of cancer cell lines. MicroRNAs (miRNAs) were purified from exosomes. Novel biomarker candidates for PDCA were identisfied from urine exosome miRNA using expression profiling, and validated in a larger number of samples using 3D digital PCR. The results of a preliminary analysis of nine PDAC and seven control subjects revealed that the miR-3940-5p/miR-8069 ratio in urine exosomes was elevated in the patients with PDAC. Experiments using cultured cancer cell lines revealed that the elevation of the miR-3940-5p/miR-8069 ratio was specific for PDAC. Furthermore, the elevation of the miR-3940-5p/miR-8069 ratio in exosomes tended to be higher in the urine than in the serum of patients with PDAC. Validation experiments on 43 PDAC, 12 chronic pancreatitis and 25 control subjects demonstrated that the
\end{abstract}

Correspondence to: Dr Kazushi Sugimoto, Department of Gastroenterology and Hepatology, Mie University Graduate School of Medicine, 2-174 Edobashi, Tsu, Mie 514-8507, Japan

E-mail:kazushi@clin.medic.mie-u.ac.jp

Abbreviations: PDAC, pancreatic ductal adenocarcinoma; CP, chronic pancreatitis; CA19-9, carbohydrate antigen 19-9; PPV, positive predictive value; NPV, negative predictive value; miRNA, microRNA

Key words: pancreatic cancer, microRNA, urine, exosome, 3D digital PCR
miR-3940-5p/miR-8069 ratio in urine exosomes was elevated in PDAC at a relatively early stage of the disease. When this ratio was used in combination with CA19-9 for the diagnosis of PDAC, the sensitivity and positive predictive value improved to 93.0 and $78.4 \%$, respectively, when either of them was positive. Additionally, the positive predictive value reached $100 \%$ when both were positive. The negative predictive value also improved to $89.7 \%$ when both were negative. The miR-3940-5p/miR-8069 ratio in urine exosomes may be useful as a tool for the diagnosis of PDAC, particularly when used in combination with CA19-9.

\section{Introduction}

Pancreatic cancer still has an extremely poor prognosis. The number of deaths due to pancreatic cancer has been increasing worldwide. In fact, it was predicted that the total deaths from pancreatic cancer will become the second leading cause of cancer-related deaths in the United States by 2030 (1). In addition, National Cancer Center Japan (https://ganjoho. jp/reg_stat/statistics/stat/summary.html) reported that pancreatic cancer became the fourth leading cause of cancer deaths in Japan in 2016. There are several reasons for the low survival rate of pancreatic cancer. Among them, the most pressing problem that has been very hard to resolve is the difficulty of diagnosing the disease at an early stage. It is very difficult to detect pancreatic cancer by imaging studies performed in routine medical examinations, and by the time it is discovered due to worsening symptoms, such as jaundice or abdominal/back pain, it would have already progressed to an incurable phase. Another problem for its early diagnosis is the lack of a useful biomarker.

Many types of biomarkers for pancreatic cancer in patient serum, pancreatic juice, or stool have been proposed. Among these biomarkers, serum carbohydrate antigen (CA) 19-9 is the most commonly used in the usual clinical setting. However, several reports have suggested that the positive predictive value (PPV) of CA19-9 for pancreatic cancer was very low $(0.5$ to $0.9 \%)$ in asymptomatic patients $(2,3)$. In addition, serum CA19-9 is elevated in many other types of cancers and benign diseases. As such, the utility of serum CA19-9 testing as a routine screening tool to find curable pancreatic cancer is 
extremely limited. Although pancreatic cancers are found at an advanced stage in the majority of cases, Yachida et al (4) have shown that the progression of pancreatic cancer is relatively slow. According to their report, it takes at least 10 years for the initiated tumor cells to become parental non-metastatic founder cells, and at least 5 more years for the acquisition of metastatic ability. This suggests that if a potent diagnostic tool that can detect early stages of the disease was available, we may have a better chance than previously thought to find and treat pancreatic cancer before it becomes fatal.

MicroRNA (miRNA) is a small non-coding RNA that binds to complementary sequences in the $3^{\prime}$ untranslated region of target messenger RNAs (mRNAs); this leads to the inhibition of their translation or their degradation, and thereby negatively regulate gene expression. Recent studies have revealed that many types of cancer cells produce specific miRNAs (5-7); therefore, the utility of specific miRNAs as biomarkers for cancers has been widely investigated. Many studies have shown that specific alterations in miRNA expression patterns are observable in the blood $(8,9)$, pancreatic tissues $(10,11)$, or pancreatic juice $(12,13)$ of pancreatic cancer patients. For example, Abue et al (9) reported that miRNA-483-3p levels were elevated in the blood of pancreatic cancer patients when compared to intraductal papillary neoplasm patients and healthy controls, and it could be used to differentiate pancreatic cancer from intraductal papillary neoplasms with a sensitivity of $43.8 \%$. In addition, Panarelli et al (10) showed that miR-21, miR-221, miR-155, miR-100, and miR-181b were overexpressed in resected pancreatic cancer tissues when compared to benign lesions.

One advantage of using miRNAs as biomarkers is their stability. Even though plasma has a high level of RNase activity, miRNAs are resistant to being degraded by them (14); they are protected from endogenous RNase activity because cells release the miRNAs by incorporating them into exosomes (15). Exosomes are membrane-bound particles of 40 to $100 \mathrm{~nm}$ that are released from many types of cells; they can be taken up by neighboring cells, and modulate the bioactivities of the recipient cells with their components, such as lipids, proteins, and nucleic acids (16). Exosomes are enriched with several hundred folds of mRNAs and miRNAs as compared to the donor cells, and the miRNAs in urinary exosomes have been widely explored as biomarkers, especially for cancers of the urinary tract (17-19). Moreover, some have raised the possibility that urine exosomes can be derived from diseases in organs other than the urinary tract $(20,21)$.

Given these facts, in the present study, we investigated miRNAs specific for pancreatic cancer in urinary exosomes, and identified a novel biomarker candidate for this disease.

\section{Materials and methods}

Patients. Patients admitted to the Gastroenterology Department of Mie University Hospital between October 2015 and February 2017 with pancreatic ductal adenocarcinoma (PDAC) or chronic pancreatitis (CP), including autoimmune pancreatitis, were enrolled in this study. The final diagnoses of PDAC were confirmed by endoscopic ultrasound-guided fine-needle aspiration, endoscopic retrograde cholangiopancreatography, or endoscopic biopsy. The diagnoses of CP were made by endoscopic ultrasound-guided fine-needle aspiration and endoscopic retrograde cholangiopancreatography. The diagnoses of autoimmune pancreatitis were made from elevated serum IgG4 levels and positive staining for IgG4 by immunohistochemistry. Healthy control subjects, adjusted by age and sex, were also enrolled. All patients were newly diagnosed and treatment-naïve. Patients with a present or past history of any type of malignant neoplasm were excluded from this study.

This study was approved by the Ethics Committee of Mie University Hospital (reference no. 2833). Written informed consent was obtained from each patient included in the study. The study protocol conformed to the ethical guidelines of the 1975 Declaration of Helsinki, as reflected in the a priori approval from the institution's human research committee.

Urine and serum sample collection. Urine samples (more than $10 \mathrm{ml}$ ) were collected from the PDAC, CP, and control subjects before highly invasive examinations, such as endoscopic procedures. The samples were kept at $4^{\circ} \mathrm{C}$, and centrifuged at $3,000 \mathrm{x}$ g for $15 \mathrm{~min}$ within $8 \mathrm{~h}$ after collection. The supernatants were divided into $6-\mathrm{ml}$ aliquots and kept at $-30^{\circ} \mathrm{C}$ until the analyses.

Serum was also collected from each subject before highly invasive examinations. The samples were kept at $-80^{\circ} \mathrm{C}$ until the analyses.

Cell lines. The human PDAC cell lines PANC-1 (RCB2095) and MIA PaCa-2 (RCB2094), human bile duct cell line HuCCT1 (RCB1960), human hepatocellular carcinoma cell line HuH-7 (RCB1942), human liver cancer cell line Hep G2 (RCB1886), and human gastric cancer cell line KATO III (RCB2088) were purchased from RIKEN BRC. The colon cancer cell line SW480 (ATCC CCL-228) was purchased from ATCC.

Cell cultures. All cell lines were cultured according to the manufacturers' instructions. Briefly, PANC-1 cells, SW480 cells, and $\mathrm{HuH}-7$ cells were cultured at $37^{\circ} \mathrm{C}$ and $5 \% \mathrm{CO}_{2}$ in Dulbecco's modified Eagle's medium (DMEM; Thermo Fisher Scientific, Inc.) supplemented with $10 \%$ fetal bovine serum (FBS; Thermo Fisher Scientific, Inc.) and 1\% penicillin-streptomycin (Thermo Fisher Scientific, Inc.). MIA PaCa-2 cells were maintained in DMEM with $10 \%$ FBS, $2.5 \%$ horse serum (Thermo Fisher Scientific, Inc.) and 1\% penicillin-streptomycin. HepG2 cells were maintained in modified Eagle's medium (Thermo Fisher Scientific, Inc.) with 10\% FBS and $1 \%$ penicillin-streptomycin. Kato III cells and HuCCT1 cells were maintained in Roswell Park Memorial Institute medium 1640 (Thermo Fisher Scientific, Inc.) containing 10\% FBS and $1 \%$ penicillin-streptomycin.

After incubation, the cells were washed with phosphate-buffered saline and incubated for $24 \mathrm{~h}$ in FBS-free medium. Then, the supernatants were centrifuged twice at $3,000 \times \mathrm{g}$ for $15 \mathrm{~min}$ and collected and divided into 6-ml aliquots and kept at $-30^{\circ} \mathrm{C}$ until the analyses.

Isolation of exosomes from urine samples and culture media. Exosomes were isolated from the urine samples and culture media using ExoQuick-TC (System Biosciences) according to 
the manufacturer's protocol. Briefly, the frozen urine samples or culture media were thawed and centrifuged at 3,000 x g for 15 min to eliminate impure substances, such as cells and cell debris. Next, $5 \mathrm{ml}$ of each supernatant and $1 \mathrm{ml}$ of ExoQuick-Tc exosome precipitation solution were mixed well by flicking the tubes. The mixtures were allowed to settle overnight (for at least $12 \mathrm{~h}$ ) at $4^{\circ} \mathrm{C}$ and were then centrifuged at 1,500 $\mathrm{x}$ g for $30 \mathrm{~min}$. The exosomes appeared as pellets and were isolated by aspiration of the supernatants.

Isolation of exosomes from serum samples. Exosomes were isolated from serum samples by using ExoQuick (System Biosciences) according to the manufacturer's instructions. The frozen sera were thawed and centrifuged at 3,500 x g for $20 \mathrm{~min}$ to eliminate impure substances, such as cells and cell debris. Next, $250 \mu \mathrm{l}$ of each supernatant and $63 \mu \mathrm{l}$ of Exo Quick exosome precipitation solution were mixed well by pipetting. The solutions were then allowed to settle for $30 \mathrm{~min}$ on ice and were then centrifuged at $1,500 \mathrm{x}$ for $35 \mathrm{~min}$. The exosomes appeared as pellets and were isolated by aspiration of the supernatants.

Purification of the miRNA from exosomes. The miRNAs were purified from the exosomes by using a miRCURY RNA Isolation kit (Qiagen). Each exosome pellet was mixed with $350 \mu \mathrm{l}$ of lysis solution by vortexing for over $15 \mathrm{sec}$ and was confirmed to have completely dissolved. Subsequently, $200 \mu 1$ of $99.5 \%$ ethanol was added to the mixture and vortexed for over $10 \mathrm{sec}$. The lysate was applied to a column assembled with a collection tube and centrifuged at $14,000 \mathrm{x} \mathrm{g}$ for $1 \mathrm{~min}$, after which the flow-through was discarded. Next, $400 \mu \mathrm{l}$ of wash solution was applied to the column, and centrifuged at $14,000 \mathrm{x}$ for $1 \mathrm{~min}$, after which the flow-through was discarded. This washing procedure was repeated twice, and the column was dried by centrifugation at $14,000 \mathrm{x}$ g for $2 \mathrm{~min}$. Afterwards, $50 \mu \mathrm{l}$ of eluent was applied to the column settled into an elution tube and centrifuged at $200 \mathrm{x} \mathrm{g}$ for $2 \mathrm{~min}$, then $14,000 \mathrm{xg}$ for $1 \mathrm{~min}$. The extraction liquid was applied to the column again and centrifuged at $200 \mathrm{x} g$ for $2 \mathrm{~min}$, followed by $14,000 \mathrm{x} \mathrm{g}$ for $1 \mathrm{~min}$. The purified samples were stored at $-80^{\circ} \mathrm{C}$.

Quality checking of the miRNA. The quality of the extracted miRNA was examined using an Agilent Bioanalyzer 2100 and an RNA 6000 Small RNA kit (Agilent Technologies). All of the miRNA samples were confirmed to be suitable for further analyses.

miRNA expression profiling. The miRNA expression profiles were examined in nine PDAC patients and seven controls with SurePrint G3 Human miRNA Microarray, 8x60K Rel. 21.0, consist of 2549 human miRNA probes (Agilent Technologies) with $60 \mathrm{ng}$ miRNA per sample according to the manufacturer's protocol. The hybridized chip was scanned using the G2539A Microarray Scanner (Agilent Technologies) and analyzed using Gene Spring GX software, version 13.1 (Agilent Technologies). Raw intensities were not normalized because the levels of miRNA expression were low.

Detection of miRNA. The levels of miRNA expression were validated in the culture media, patient sera, and a large number of urine samples using 3D digital PCR according to the results of the microarray.

Complementary DNA (cDNA) was synthesized from $6 \mu \mathrm{l}$ of miRNA solution by using the miRCURY Universal cDNA Synthesis kit II (Qiagen) with the following conditions according to the manufacturer's instructions: $42^{\circ} \mathrm{C}$ for $60 \mathrm{~min}, 95^{\circ} \mathrm{C}$ for $5 \mathrm{~min}$, and then held at $4^{\circ} \mathrm{C}$. Digital PCR was performed using a QuantStudio ${ }^{\circledR}$ 3D digital PCR (Thermo Fisher Scientific, Inc.). The reactions were prepared in a final volume of $18 \mu \mathrm{l}$, containing $9 \mu \mathrm{l}$ of Mastermix QuantStudio ${ }^{\circledR}$ 3D digital PCR (Thermo Fisher Scientific, Inc.), $0.72 \mu 1$ of cDNA, $1.8 \mu \mathrm{l}$ of primers from the miRCURY LNA PCR Primer Set (Qiagen), and $1.8 \mu$ l of SYBR ${ }^{\circledR}$ Green I dye (Thermo Fisher Scientific, Inc.). The working solution of SYBR ${ }^{\circledR}$ Green I dye was diluted $(1: 1,000)$ with $100 \%$ dimethyl sulfoxide and stored in aliquots at $-20^{\circ} \mathrm{C}$. The diluted SYBR ${ }^{\circledR}$ Green I dye was further diluted (1:20) with Tris-EDTA buffer ( $\mathrm{pH} \mathrm{8.0)}$. The reaction $\operatorname{mix}(14.5 \mu \mathrm{l}$ out of $18 \mu \mathrm{l})$ was loaded onto QuantStudio 3D digital PCR chips by using a QuantStudio 3D digital PCR chip loader. The amplification was performed with the following conditions: $96^{\circ} \mathrm{C}$ for $10 \mathrm{~min}, 39$ cycles of $60^{\circ} \mathrm{C}$ for $2 \mathrm{~min}$ and $98^{\circ} \mathrm{C}$ for $30 \mathrm{sec}, 60^{\circ} \mathrm{C}$ for $2 \mathrm{~min}$, and then holding at $10^{\circ} \mathrm{C}$. The amplification results were analyzed with QuantStudio $^{\circledR}$ 3D AnalysisSuite ${ }^{\mathrm{TM}}$ Cloud Software (Applied Biosystems). The software assessed the quality of the chip data and estimated the concentrations by counting the number of positive chambers.

Statistical analysis. All statistical tests were analyzed using SPSS software, version 24.0 (IBM Inc.). The Fisher exact test was applied to test the categorical data, and the Wilcoxon rank-sum test or Kruskal-Wallis test with a Dunn-Bonferroni post hoc test was applied to test the continuous data. The accuracy was investigated using receiver operating characteristic (ROC) curve analysis, the area under the curve (AUC), and accuracy measures for determining a suitable cutoff value. $\mathrm{P}<0.05$ was considered to indicate a statistically significant difference.

\section{Results}

Identification of the miR-3940-5p/miR-8069 ratio as a novel PDAC biomarker. Firstly, as a preliminary study, we analyzed the expression of miRNA in urine exosomes by microarray in nine PDAC and seven control subjects, and the expression profiles were compared between these groups to find a candidate PDAC biomarker. Table I shows the characteristics of the subjects. There was no significant difference in the characteristics (age and sex) between the PDAC and control groups. In total, 38 miRNAs were upregulated by more than two-fold in the PDAC patients when compared to the controls. The five most upregulated miRNAs in the PDAC group are listed in Table II. Of note, the expression level of miR-3940-5p was significantly greater in all of the PDAC patients than in the controls (fold change: 3.047; $\mathrm{P}=0.036$ ). On the other hand, miR-8069 was expressed at a similar level in all of the PDAC patients and controls (data not shown). Based on these results, we identified the miR-3940-5p/miR-8069 ratio in urine exosomes as a candidate novel biomarker for PDAC. 
Table I. Patient characteristics analyzed by the microarray.

\begin{tabular}{|c|c|c|c|c|c|c|}
\hline Subjects & $\begin{array}{l}\text { Age, } \\
\text { years }\end{array}$ & Sex & $\begin{array}{l}\text { Size } \\
\mathrm{mm}\end{array}$ & $\begin{array}{l}\text { Tumor } \\
\text { location }\end{array}$ & $\begin{array}{l}\text { T classification } \\
\text { (UICC 8th) }\end{array}$ & $\begin{array}{l}\text { Clinical stage } \\
\text { (UICC 8th) }\end{array}$ \\
\hline \multirow[t]{9}{*}{ Patients with pancreatic ductal adenocarcinoma } & 65 & $\mathrm{M}$ & 18 & Head & $\mathrm{T} 1$ & III \\
\hline & 83 & M & 22 & Tail & $\mathrm{T} 2$ & II \\
\hline & 76 & $\mathrm{~F}$ & 22 & Head & $\mathrm{T} 4$ & III \\
\hline & 53 & $\mathrm{~F}$ & 40 & Head & $\mathrm{T} 4$ & III \\
\hline & 73 & $\mathrm{~F}$ & 30 & Head & $\mathrm{T} 4$ & IV \\
\hline & 84 & M & 16 & Body-tail & $\mathrm{T} 1$ & I \\
\hline & 67 & $\mathrm{M}$ & 20 & Head & $\mathrm{T} 2$ & I \\
\hline & 89 & $\mathrm{M}$ & 27 & Head & $\mathrm{T} 2$ & III \\
\hline & 59 & $\mathrm{~F}$ & 46 & Body-tail & $\mathrm{T} 4$ & IV \\
\hline \multirow[t]{7}{*}{ Controls } & 78 & $\mathrm{M}$ & & & & \\
\hline & 62 & $\mathrm{M}$ & & & & \\
\hline & 76 & $\mathrm{~F}$ & & & & \\
\hline & 68 & $\mathrm{~F}$ & & & & \\
\hline & 57 & $\mathrm{M}$ & & & & \\
\hline & 81 & $\mathrm{~F}$ & & & & \\
\hline & 76 & M & & & & \\
\hline
\end{tabular}

UICC 8th, the eighth edition of the International Union Against Cancer classification; M, male: F, female.

Table II. Top five most upregulated microRNAs in pancreatic ductal adenocarcinoma.

\begin{tabular}{llcc}
\hline microRNA & \multicolumn{1}{c}{ Sequence } & $\begin{array}{c}\text { Fold } \\
\text { change }\end{array}$ & P-value \\
\hline hsa-miR-3940-5p & CAGAGCCCGCCC & 3.047 & 0.036 \\
hsa-miR-6085 & TGTGCTCCCCCAGC & 2.973 & 0.108 \\
hsa-miR-4516 & GCCCCGACCCTTC & 2.662 & 0.120 \\
hsa-miR-4298 & CTGCCTCCTCCTCC & 2.623 & 0.061 \\
hsa-miR-6749-5p & GCTCCCCCAACCC & 2.527 & 0.184 \\
\hline
\end{tabular}

miR, microRNA.

Preliminary validation in cell lines. As the next step, we attempted to determine whether the increase in the miR-3940-5p/miR-8069 ratio was specific for PDAC by using seven different cancer cell lines. Because the expression levels of miRNA in the exosomes, especially those excreted into urine, were very low, it was difficult to quantify them accurately with the conventional quantitative PCR method; therefore, we measured them by 3D digital PCR. As shown in Fig. 1, the miR-3940-5p/miR-8069 ratio in exosomes from the culture media was significantly higher in the two PDAC cell lines (PANC-1 and MIA PaCa-2) than in the non-pancreatic cancer cell lines (HuH-7, HepG2, SW480, HuCCT1, and Kato III; $\mathrm{P}<0.001)$, suggesting that the increase in the miR-3940-5p/miR-8069 ratio was specific to PDAC.

Comparison of the expression levels in serum and urine exosomes. Next, we analyzed the miR-3940-5p/miR-8069 ratio in exosomes derived from the serum or urine of seven PDAC patients. Fig. 2 shows that the miR-3940-5p/miR-8069 ratio was greater in the urine exosomes than in the serum exosomes, although the difference did not reach a statistically significant level, perhaps due to the small sample size. These results suggested that urine samples are more suitable than serum samples for analyzing the miR-3940-5p/miR-8069 ratio as a biomarker for PDAC.

Validation in a larger number of samples. According to the results of the preliminary analyses, we attempted to further validate the usefulness of the miR-3940-5p/miR-8069 ratio in urine exosomes as a biomarker for PDAC in a larger number of samples. We examined a total of 43 PDAC, $12 \mathrm{CP}$, and 25 control subjects. Table III shows the characteristics of the patients. The mean tumor size of the PDAC cases was relatively large at $33.0 \pm 16.3 \mathrm{~mm}$. According to the eighth edition of the International Union Against Cancer (UICC) classification system, $44.2 \%$ of the PDAC patients had T4 tumors, and $46.5 \%$ had clinical stage IV disease. As was mentioned above, the PDAC group in our study included advanced cases of PDAC.

The miR-3940-5p/miR-8069 ratio in the urine exosomes of each group is shown in Fig. 3. The median values of the control, CP, and PDAC subjects were $0.47,0.54$, and 1.07 , respectively. The $\mathrm{miR}-3940-5 \mathrm{p} / \mathrm{miR}-8069$ ratio in urine exosomes was significantly higher in the PDAC group than in the control group $(\mathrm{P}<0.001)$ or the $\mathrm{CP}$ group $(\mathrm{P}<0.001)$.

To clarify the stage of PDAC at which the miR-3940$5 \mathrm{p} / \mathrm{miR}-8069$ ratio begins to increase, we analyzed the ratio according to $\mathrm{T}$ classification and clinical stage as defined by the eighth edition of the UICC classification (Fig. 4A and B). As shown in the graphs, the miR-3940-5p/miR-8069 ratio in the urine exosomes was significantly higher in the PDAC 


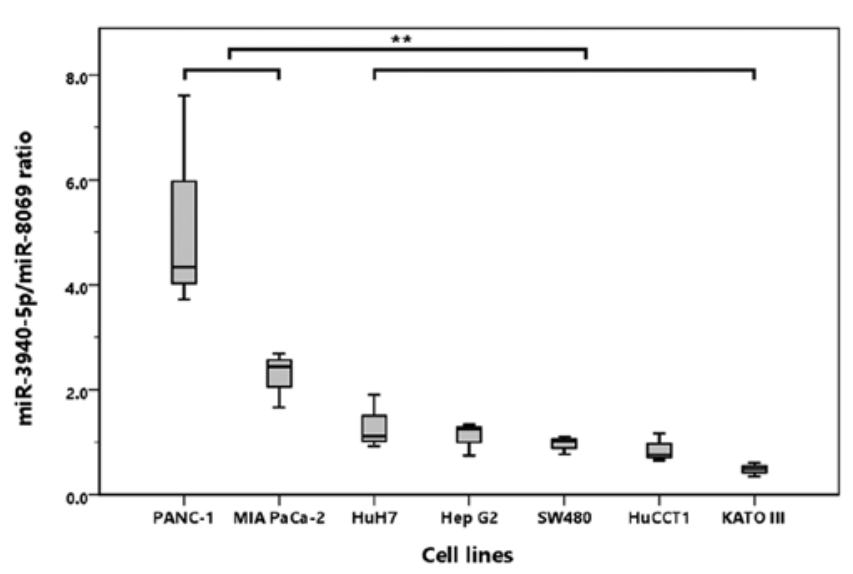

Figure 1. miR-3940-5p/miR-8069 ratio in exosomes from cancer cell lines. The levels of microRNAs in exosomes isolated from culture media were measured. The miR-3940-5p/miR-8069 ratio was significantly greater in pancreatic cancer cell lines than in other cancer cell lines. ${ }^{* *} \mathrm{P}<0.01$. miR, microRNA.

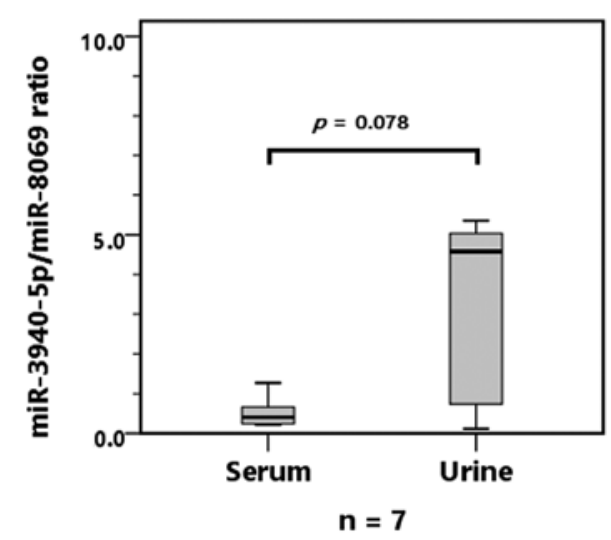

Figure 2. Comparison of the miR-3940-5p/miR-8069 ratio in exosomes derived from the serum and urine of patients with pancreatic ductal adenocarcinoma. The miR-3940-5p/miR-8069 ratio was greater in urine exosomes than in serum exosomes. miR, microRNA.

patients with T4 tumors or stage IV disease than in the control subjects. Interestingly, the ratio also tended to be elevated in $\mathrm{T} 1$ or stage I PDAC patients, although the differences did not reach a statistically significant level, perhaps due to the small sample sizes. Nevertheless, these results suggest the possibility that the miR-3940-5p/miR-8069 ratio in urine exosomes starts to increase from a relatively early stage of PDAC.

Diagnostic value of the miR-3940-5p/miR-8069 ratio for $P D A C$. The diagnostic accuracy of using the $\mathrm{miR}-3940-5 \mathrm{p} / \mathrm{miR}-$ 8069 ratio in urine exosomes for differentiating PDAC from $\mathrm{CP}$ and the controls was also evaluated using the receiver operating characteristic (ROC) curve (Fig. 5). The AUC was 0.732 (95\% confidence interval: 0.621-0.843). The cutoff point established by the ROC curve was 0.939. By using this cutoff, the sensitivity, specificity, PPV, and negative predictive value (NPV) for PDAC were 58.1, 89.2, 86.2, and 64.7\%, respectively (Table IV).

Diagnostic value of the miR-3940-5p ratio with CA19-9. Finally, we examined the utility of the miR-3940-5p/miR-8069 ratio in
Table III. Background characteristics of the 43 pancreatic ductal adenocarcinoma cases.

\begin{tabular}{lcc}
\hline Characteristics & Number/mean \pm SD & Percentage \\
\hline Age, years & $68.4 \pm 10.2$ & \\
Sex (male) & 25 & 58.1 \\
Tumor location & & \\
Head & 26 & 60.5 \\
Body-tail & 17 & 39.5 \\
Tumor size, mm & $33.0 \pm 16.3$ & \\
T classification (UICC 8th) & & \\
T1 & 6 & 14.0 \\
T2 & 13 & 30.2 \\
T3 & 5 & 11.6 \\
T4 & 19 & 44.2 \\
Clinical stage (UICC 8th) & & \\
I & 12 & 27.9 \\
II & 3 & 7.0 \\
III & 8 & 18.6 \\
IV & 20 & 46.5 \\
\hline
\end{tabular}

UICC 8th, the eighth edition of the International Union Against Cancer classification.

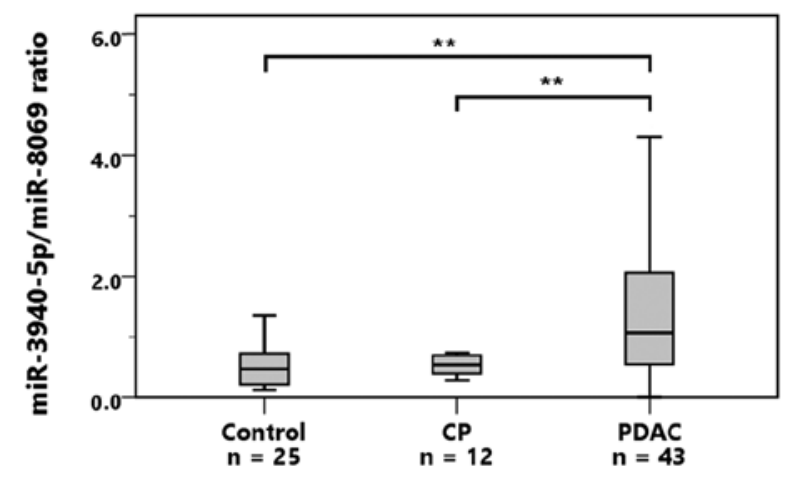

Figure 3. miR-3940-5p/miR-8069 ratio in the urine exosomes of the study subjects. The miR-3940-5p/miR-8069 ratio in urine exosomes was compared among 25 control, $12 \mathrm{CP}$ and $43 \mathrm{PDAC}$ samples. The ratio was significantly greater in the PDAC samples than in the control or CP samples. The mean values for the control, $\mathrm{CP}$ and PDAC samples were $0.47,0.54$ and 1.07 , respectively. ${ }^{* *} \mathrm{P}<0.01$. CP, chronic pancreatitis; miR, microRNA; PDAC, pancreatic ductal adenocarcinoma.

urine exosomes in combination with CA19-9 as biomarkers for differentiating PDAC. In our study subjects, the sensitivity, specificity, PPV, and NPV of CA19-9 (cutoff: $37 \mathrm{U} / \mathrm{ml}$ ) were 79.1, 81.1, 82.9, and 76.9\%, respectively (Table IV). After the miR-3940-5p ratio and CA19-9 were combined, when either of them was positive, the sensitivity and PPV reached 93.0 and $78.4 \%$, respectively. In addition, the PPV reached $100 \%$ when both were positive. In contrast, when both were negative, the specificity and NPV were 70.3 and $89.7 \%$, respectively (Table IV). These results clearly indicated that a combined analysis of the miR-3940-5p/miR-8069 ratio in urine exosomes and CA19-9 is more helpful for predicting or ruling out PDAC than when they are used alone. 
Table IV. Diagnostic ability of the miR-3940-5p/miR-8069 ratio and CA19-9 for pancreatic ductal adenocarcinoma.

\begin{tabular}{lccc}
\hline Parameters & miR-3940-5p/miR-8069 & CA19-9 & miR-3940-5p/miR-8069+ CA19-9 \\
\hline Sensitivity, \% & 58.1 & 79.1 & $93.0^{\mathrm{a}}$ \\
Specificity, \% & 89.2 & 81.1 & $70.3^{\mathrm{b}}$ \\
Positive predictive value, $\%$ & 86.2 & 82.9 & $78.4^{\mathrm{a}}\left(100^{\mathrm{c}}\right)$ \\
Negative predictive value, $\%$ & 64.7 & 76.9 & $89.7^{\mathrm{b}}$ \\
\hline
\end{tabular}

${ }^{\mathrm{a} A t}$ least one of these parameters is positive; ${ }^{\mathrm{b} B o t h}$ parameters are negative; ${ }^{\mathrm{B}}$ Both parameters are positive. CA19-9, carbohydrate antigen $19-9$; miR, microRNA.

\section{A}

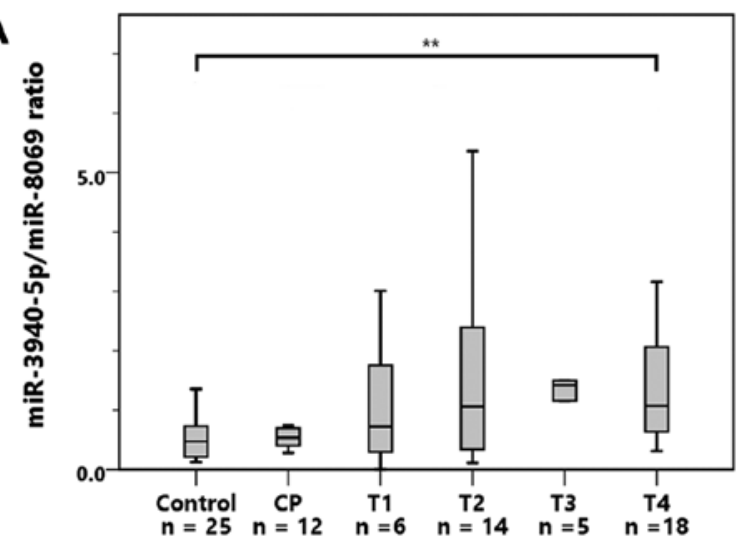

B

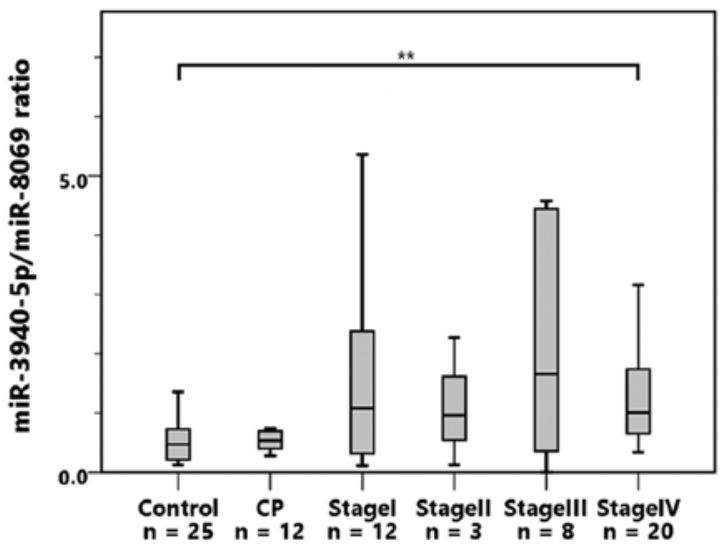

Figure 4. Alterations in the miR-3940-5p/miR-8069 ratio in urine exosomes at different stages of PDAC. The miR-3940-5p/miR-8069 ratio was analyzed according to (A) T classification and (B) clinical stage as defined by the eighth edition of the International Union Against Cancer classification. The miR-3940-5p/miR-8069 ratio tended to increase from the early stages of PDAC. ${ }^{* *} \mathrm{P}<0.01$. CP, chronic pancreatitis; miR, microRNA; PDAC, pancreatic ductal adenocarcinoma.

\section{Discussion}

In the present study, we identified the $\mathrm{miR}-3940-5 \mathrm{p} / \mathrm{miR}-8069$ ratio in urine exosomes as a novel biomarker for PDAC. We also found that an elevation of this ratio was specific for PDAC in cultured cancer cell lines, and the ratio was greater in the urine exosomes than in the serum exosomes of PDAC patients. In addition, the diagnostic ability of the miR-3940-5p/miR-8069 ratio and CA19-9 value for identifying PDAC was much better when used in combination than when used alone.

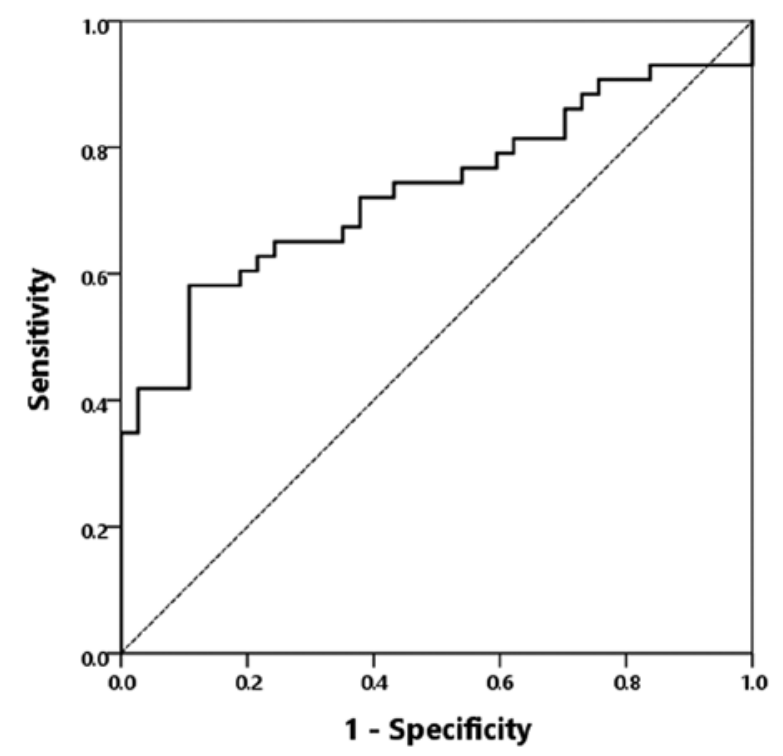

Figure 5. Receiver operating characteristic curve analysis of the miR-3940-5p/miR-8069 ratio. The area under the curve was 0.732 (95\% CI, 0.621-0.843), and the cut-off established by the receiver operating characteristic analysis was 0.939 . miR, microRNA.

Despite the development of several therapeutic options, the prognosis of PDAC is still poor. One of the biggest reasons for this is the difficulty of diagnosing the disease at an early stage. In addition to imaging studies, several biomarkers are currently used for the diagnosis of PDAC. Among them, CA19-9 is the most extensively studied biomarker, and it is known to correlate with prognosis (22); however, CA19-9 is also occasionally elevated in benign hepatobiliary diseases, pancreatitis, or other tumors, such as colorectal cancer and ovarian tumors $(23,24)$, and the PPV in asymptomatic patients is extremely low $(2,3)$. Therefore, many studies have attempted to find new diagnostic markers for PDAC in blood or pancreatic juice, and many studies have been focusing on miRNAs (8-13). In the present study, we explored miRNAs in urine exosomes, and identified the $\mathrm{miR}-3940-5 \mathrm{p} / \mathrm{miR}-8069$ ratio as a novel biomarker for PDAC. There were two major reasons why we focused on urine exosomes. First, urine is easy to obtain repeatedly from patients without any invasive procedures; as such, we believe more and more methodologies using urine samples will be developed hereafter for the diagnosis of many kinds of diseases. Indeed, recent studies have suggested the possibility that urine miRNAs can be biomarkers for several cancers, including PDAC $(25,26)$. 
The second reason is that miRNAs are more stable and concentrated in exosomes than in host cells or serum. The results of preliminary analyses using microarray data suggested that miR-3940-5p was upregulated in the urine exosomes of most PDAC patients when compared to the controls.

The precise mechanism by which exosomes derived from cells other than urinary tract are excreted into urine is not clear yet. However, several studies have actually shown that some disease-specific exosomes can be detected in urine, and they suggested that the miRNA contained in the exosomes can act as biomarkers for non-urologic diseases, including cancers $(20,21,27)$. In addition, Weber et al (28) showed the differences of miRNA expression profiles in different human body fluids, and some miRNAs showed higher expression levels in urine compared to serum. However, the amounts of urinary exosomes and the miRNAs encapsulated in them are extremely low, so it has been difficult to obtain and assess a sufficient amount of them for use as biomarkers. To overcome this problem, Yasui et al (27) developed a new nanowire-based methodology to collect miRNAs encapsulated in urine extracellular vesicles, including exosomes, microvesicles, and apoptotic bodies. Using this method, they found that many miRNAs in urine extracellular vesicles were overexpressed or downregulated in lung, liver, and pancreatic cancers (27). In our present study, we used 3D digital PCR to accurately quantify the low levels of miRNA for validation in a large number of samples. Digital PCR, which is a third generation of PCR, has increased precision and sensitivity for detecting low amounts of target copies when compared to conventional quantitative real-time PCR $(29,30)$. As such, it can be used for the detection and quantification of low levels of pathogens, rare genetic sequences, and cancer-related miRNAs (31-33). To more precisely compare the expression levels of miR-3940-5p in a large number of samples, we normalized the miR-3940-5p levels to the miR-8069 levels, because the results of microarray analysis indicated that miR-8069 was expressed at a similar level in all samples, including PDAC and control samples. We found that the miR-3940-5p/miR-8069 ratio in urine exosomes was significantly elevated in PDAC patients when compared to the controls or CP patients. We also found that this elevation could be observed at a relatively early stage of PDAC.

Sun et al (34) reported that the expression of miR-3940-5p was downregulated in non-small cell lung carcinoma (NSCLC) tissues when compared to normal lung tissues or tumor-adjacent tissues. They also found that miR-3940-5p was specifically reduced in nuclear cell proliferation antigen Ki-67-positive NSCLC cases (34). In addition, Ren et al (35) reported that miR-3940-5p may target cyclin D1 and ubiquitin specific peptide-28 to inhibit the growth of NSCLC. In the present study, the miR-3940-5p/miR-8069 ratio was increased in the PDAC group, and experimental results from studies using cultured cancer cell lines showed that this increase was specific to PDAC. However, the biological relevance of its upregulation and the reason for the discrepancy in results between PDAC and NSCLC remain to be elucidated. Furthermore, we found that the miR-3940-5p/miR-8069 ratio was greater in urine exosomes than in serum exosomes. The reason for this remains unclear, however, these results suggests that although the amount of PDAC-related exosomes that have a high miR-3940-5p/miR-8069 ratio in serum is extremely low, and a small proportion of them is excreted into the urine, the concentration of these exosomes is still higher in urine than in blood.

One of the most important findings of this study is that the miR-3940-5p/miR-8069 ratio and CA19-9 value as biomarkers can better predict PDAC when used in combination than when used alone. In fact, when we applied the cutoff point of 0.939 , which was established from the ROC curve, for the miR-3940-5p/miR-8069 ratio, and the widely clinically used cutoff of $37 \mathrm{U} / \mathrm{ml}$ for CA19-9, the sensitivity and PPV improved to 93.0 and $78.4 \%$, respectively, when either of them was positive. Moreover, the PPV reached $100 \%$ when they were both positive. Similarly, the NPV also improved to $89.7 \%$ when both were negative.

We acknowledge that this study has some limitations. First, it was carried out with a small number of samples. To improve the reliability of the findings of this study, more analyses with a larger number of patients is needed. Second, we did not clarify the relationship between the miR-3940-5p/miR-8069 ratio in urine exosomes and the prognosis of PDAC, nor possible changes in the ratio after therapy for PDAC. Although these points were beyond the scope of this study, further investigations focusing on these points are necessary as the next step in examining the miR-3940-5p/miR-8069 ratio in urine exosomes as a biomarker for PDAC.

In conclusion, the results of the present study showed that the miR-3940-5p/miR-8069 ratio in urine exosomes is elevated in PDAC patients, suggesting that it may be a potent diagnostic tool for PDAC, especially in combination with CA19-9.

\section{Acknowledgements}

The authors would like to thank Ms. Mina Tenpaku (Department of Gastroenterology and Hepatology, Mie University Graduate School of Medicine, Mie, Japan) for her technical assistance.

\section{Funding}

No funding was received.

\section{Availability of data and materials}

The datasets used and/or analyzed during the current study are available from the corresponding author on reasonable request.

\section{Authors' contributions}

KS and MT conceived and designed the study. NY, HI and MU recruited the subjects, and collected and analyzed the data. NY, YI, MT and MIk performed experiments. KS, NY and MT wrote the manuscript. MIt and YT interpreted the data and supervised the study. MIk, HI, MU, MIt and YT revised the manuscript. All authors read and approved the final manuscript.

\section{Ethics approval and consent to participate}

The present study was approved by the Ethics Committee of Mie University Hospital (reference no. 2833). Written informed consent was obtained from each patient included in the present study. 


\section{Patient consent for publication}

All participants provided consent for publication.

\section{Competing interest}

The authors declare that they have no competing interests.

\section{References}

1. Rahib L, Smith BD, Aizenberg R, Rosenzweig AB, Fleshman JM and Matrisian LM: Projecting cancer incidence and deaths to 2030: The unexpected burden of thyroid, liver, and pancreas cancers in the United States. Cancer Res 74: 2913-2921, 2014.

2. Kim JE, Lee KT, Lee JK, Paik SW, Rhee JC and Choi KW: Clinical usefulness of carbohydrate antigen 19-9 as a screening test for pancreatic cancer in an asymptomatic population. J Gastroenterol Hepatol 19: 182-186, 2004.

3. Chang CY, Huang SP, Chiu HM, Lee YC, Chen MF and Lin JT: Low efficacy of serum levels of CA 19-9 in prediction of malignant diseases in asymptomatic population in Taiwan. Hepatogastroenterology 53: 1-4, 2006.

4. Yachida S, Jones S, Bozic I, Antal T, Leary R, Fu B, Kamiyama M, Hruban RH, Eshleman JR, Nowak MA, et al: Distant metastasis occurs late during the genetic evolution of pancreatic cancer. Nature 467: 1114-1117, 2010.

5. Michael MZ, O' Connor SM, van Holst Pellekaan NG, Young GP and James RJ: Reduced accumulation of specific microRNAs in colorectal neoplasia. Mol Cancer Res 1: 882-891, 2003.

6. Iorio MV, Ferracin M, Liu CG, Veronese A, Spizzo R, Sabbioni S, Magri E, Pedriali M, Fabbri M, Campiglio M, et al: MicroRNA gene expression deregulation in human breast cancer. Cancer Res 65: 7065-7070, 2005.

7. Johnson SM, Grosshans H, Shingara J, Byrom M, Jarvis R, Cheng A, Labourier E, Reinert KL, Brown D and Slack FJ: RAS is regulated by the let-7 microRNA family. Cell 120: 635-647, 2005.

8. Li A, Omura N, Hong SM, Vincent A, Walter K, Griffith M, Borges $M$ and Goggins M: Pancreatic cancers epigenetically silence SIP1 and hypomethylate and overexpress miR-200a/200b in association with elevated circulating miR-200a and miR-200b levels. Cancer Res 70: 5226-5237, 2010.

9. Abue M, Yokoyama M, Shibuya R, Tamai K, Yamaguchi K, Sato I, Tanaka N, Hamada S, Shimosegawa T, Sugamura K and Satoh K: Circulating miR-483-3p and miR-21 is highly expressed in plasma of pancreatic cancer. Int J Oncol 46: 539-547, 2015.

10. Panarelli NC, Chen YT, Zhou XK, Kitabayashi N and Yantiss RK: MicroRNA expression aids the preoperative diagnosis of pancreatic ductal adenocarcinoma. Pancreas 41: 685-690, 2012.

11. Hong TH and Park IY: MicroRNA expression profiling of diagnostic needle aspirates from surgical pancreatic cancer specimens. Ann Surg Treat Res 87: 290-297, 2014.

12. Sadakari Y, Ohtsuka T, Ohuchida K, Tsutsumi K, Takahata S, Nakamura M, Mizumoto K and Tanaka M: MicroRNA expression analyses in preoperative pancreatic juice samples of pancreatic ductal adenocarcinoma. JOP 11: 587-592, 2010.

13. Wang J, Raimondo M, Guha S, Chen J, Diao L, Dong X, Wallace MB, Killary AM, Frazier ML, Woodward TA, et al: Circulating microRNAs in pancreatic juice as candidate biomarkers of pancreatic cancer. J Cancer 5: 696-705, 2014.

14. Mitchell PS, Parkin RK, Kroh EM, Fritz BR, Wyman SK, Pogosova-Agadjanyan EL, Peterson A, Noteboom J, O'Briant KC, Allen A, et al: Circulating microRNAs as stable blood-based markers for cancer detection. Proc Natl Acad Sci USA 105: 10513-10518, 2008.

15. Valadi H, Ekström K, Bossios A, Sjöstrand M, Lee JJ and Lötvall JO: Exosome-mediated transfer of mRNAs and microRNAs is a novel mechanism of genetic exchange between cells. Nat Cell Biol 9: 654-659, 2007.

16. Zhang J, Li S, Li L, Li M, Guo C, Yao J and Mi S: Exosome and exosomal microRNA: Trafficking, sorting, and function. Genomics Proteomics Bioinformatics 13: 17-24, 2015.

17. Mlcochova H, Hezova R, Stanik $M$ and Slaby O: Urine microRNAs as potential noninvasive biomarkers in urologic cancers. Urol Oncol 32: 41.e1-e9, 2014.
18. Øverbye A, Skotland T, Koehler CJ, Thiede B, Seierstad T, Berge V, Sandvig K and Llorente A: Identification of prostate cancer biomarkers in urinary exosomes. Oncotarget 6: 30357-30376, 2015.

19. Franzen CA, Blackwell RH, Foreman KE, Kuo PC, Flanigan RC and Gupta GN: Urinary exosomes: The potential for biomarker utility, intercellular signaling and therapeutics in urological malignancy. J Urol 195: 1331-1339, 2016.

20. Conde-Vancells J, Rodriguez-Suarez E, Gonzalez E, Berisa A, Gil D, Embade N, Valle M, Luka Z, Elortza F, Wagner C, et al: Candidate biomarkers in exosome-like vesicles purified from rat and mouse urine samples. Proteomics Clin Appl 4: 416-425, 2010.

21. Gildea JJ, Carlson JM, Schoeffel CD, Carey RM and Felder RA: Urinary exosome miRNome analysis and its applications to salt sensitivity of blood pressure. Clin Biochem 46: 1131-1134, 2013.

22. Ballehaninna UK and Chamberlain RS: The clinical utility of serum CA 19-9 in the diagnosis, prognosis and management of pancreatic adenocarcinoma: An evidence based appraisal. J Gastrointest Oncol 3: 105-119, 2012.

23. Stiksma J, Grootendorst DC and van der Linden PW: CA 19-9 as a marker in addition to CEA to monitor colorectal cancer. Clin Colorectal Cancer 13: 239-244, 2014.

24. Pandey D, Sharma R, Sharma S and Salhan S: Unusually high serum levels of CA 19-9 in an ovarian tumour: Malignant or benign? J Clin Diagn Res 11: QD08-QD10, 2017.

25. Debernardi S, Massat NJ, Radon TP, Sangaralingam A, Banissi A, Ennis DP, Dowe T, Chelala C, Pereira SP, Kocher HM, et al: Noninvasive urinary miRNA biomarkers for early detection of pancreatic adenocarcinoma. Am J Cancer Res 5: 3455-3466, 2015.

26. Kao HW, Pan CY, Lai CH, Wu CW, Fang WL, Huang KH and Lin WC: Urine miR-21-5p as a potential non-invasive biomarker for gastric cancer. Oncotarget 8: 56389-56397, 2017.

27. Yasui T, Yanagida T, Ito S, Konakade Y, Takeshita D, Naganawa T, Nagashima K, Shimada T, Kaji N, Nakamura Y, et al: Unveiling massive numbers of cancer-related urinary-microRNA candidates via nanowires. Sci Adv 3: e1701133, 2017.

28. Weber JA, Baxter DH, Zhang S, Huang DY, Huang KH, Lee MJ, Galas DJ and Wang K: The microRNA spectrum in 12 body fluids. Clin Chem 56: 1733-1741, 2010.

29. Brunetto GS, Massoud R, Leibovitch EC, Caruso B, Johnson K, Ohayon J, Fenton K, Cortese I and Jacobson S: Digital droplet PCR (ddPCR) for the precise quantification of human T-lymphotropic virus 1 proviral loads in peripheral blood and cerebrospinal fluid of HAM/TSP patients and identification of viral mutations. J Neurovirol 20: 341-351, 2014.

30. Zhao S, Lin H, Chen S, Yang M, Yan Q, Wen C, Hao Z, Yan Y, Sun Y, Hu J, et al: Sensitive detection of Porcine circovirus-2 by droplet digital polymerase chain reaction. J Vet Diagn Invest 27: 784-788, 2015.

31. Pekin D, Skhiri Y, Baret JC, Le Corre D, Mazutis L, Salem CB, Millot F, El Harrak A, Hutchison JB, Larson JW, et al: Quantitative and sensitive detection of rare mutations using droplet-based microfluidics. Lab Chip 11: 2156-2166, 2011.

32. Gutiérrez-Aguirre I, Rački N, Dreo T and Ravnikar M: Droplet digital PCR for absolute quantification of pathogens. Methods Mol Biol 1302: 331-347, 2015.

33. Conte D, Verri C, Borzi C, Suatoni P, Pastorino U, Sozzi G and Fortunato O: Novel method to detect microRNAs using chip-based QuantStudio 3D digital PCR. BMC Genomics 16: 849,2015

34. Sun Y, Su B, Zhang P, Xie H, Zheng H, Xu Y, Du Q, Zeng H, Zhou X, Chen C and Gao W: Expression of miR-150 and miR-3940-5p is reduced in non-small cell lung carcinoma and correlates with clinicopathological features. Oncol Rep 29: 704-712, 2013.

35. Ren K, Li Y, Lu H, Li Z and Han X: miR-3940-5p functions as a tumor suppressor in non-small cell lung cancer cells by targeting cyclin D1 and ubiquitin specific peptidase-28. Transl Oncol 10: 80-89, 2017.

This work is licensed under a Creative Commons Attribution-NonCommercial-NoDerivatives 4.0 International (CC BY-NC-ND 4.0) License. 